\title{
Internationale Klimagerechtigkeit - Wieso es nicht allein auf die Staaten ankommt
}

\section{Klimawandel als ethische Frage in den internationalen Beziehungen}

Kann die naturwissenschaftliche Klimaforschung zu den Ursachen und Folgen der globalen Erwärmung Auskunft geben und die wissenschaftlichen Grundlagen für die Formulierung klimapolitischer Ziele liefern, so ist die Frage, was in Bezug auf den Klimawandel getan werden soll, grundsätzlich dem Feld der Ethik zuzurechnen (Broome 2008). Ethische Erwägungen und Fragen der Gerechtigkeit spielen in der internationalen Klimapolitik daher auch von Anfang an eine zentrale Rolle. Grob lassen sich hier drei zentrale Themenfelder identifizieren, bei denen ethische Fragestellungen von Bedeutung sind:

1. Bereits die Festlegung auf ein klimapolitisches Ziel birgt ethische Implikationen. Bei der Klimakonferenz der Vereinten Nationen (VN) in Cancún Ende 2010 einigte sich die internationale Staatengemeinschaft darauf, die vom Menschen verursachte Erderwärmung im Vergleich zum vorindustriellen Niveau auf $2^{\circ} \mathrm{C}$ begrenzen zu wollen. Dieses Ziel kann bereits als äußerst anspruchsvoll gelten, da es erfordert, dass die globalen Emissionen innerhalb der nächsten 10 Jahre ihren Scheitelpunkt erreichen und danach kontinuierlich stark sinken (Rogelj u. a. 2011). Gleichzeitig drängen Staaten, die von den Auswirkungen des Klimawandels besonders getroffen werden, allen voran die kleinen Inselstaaten, auf eine Korrektur dieses Werts auf $1,5^{\circ} \mathrm{C}$, da bereits der mit einer Erwärmung um $2^{\circ} \mathrm{C}$ einhergehende Meeresspiegelanstieg ihre Existenz bedroht. Wie ist es ethisch zu bewerten, dass beim aktuellen Ziel der internationalen Klimapolitik der Fortbestand ganzer Staaten bedroht ist?

2. Eine weitere ethische Dimension der Klimaproblematik betrifft den Umgang mit den Auswirkungen der nicht verhinderten oder nicht mehr zu verhindernden Erwärmung, also die Anpassung an den Klimawandel sowie die Kompensation für Klimaschäden. Durch Folgen wie verminderte Nahrungsmittelproduktion und Wasserverfügbarkeit, den Anstieg des Meeresspiegels und den Einbruch der wirtschaftlichen Leistungsfähigkeit unterminiert Klimawandel die Lebensgrundlagen von Menschen in nahezu allen Weltregionen (Sommer 2011: 60 f.). Regionen und Länder, die in besonderem Ausmaß hiervon betroffen sein werden, wie etwa zahlreiche Staaten Afrikas südlich der Sahara, verfügen häufig über eine äußerst geringe An- 
passungsfähigkeit. Gleichzeitig haben diese Staaten aufgrund ihres technologischen und wirtschaftlichen Entwicklungsniveaus - historisch und aktuell - kaum zur Verursachung des Problems beigetragen.

3. Im Zentrum aller ethischen Erwägungen der Klimaproblematik steht aber die Frage, wie zwischen den Staaten die Lasten verteilt werden sollen, um die globale Allmende eines stabilen Klimas zu schützen (Gardiner 2004: 579; Harris 2010: 10). Grundsätzlich besteht sowohl unter Klimaethikern als auch den Staaten der Welt bezüglich dieser Frage ein weitgehender Konsens: den frühindustrialisierten OECD-Staaten, die historisch stark zur Verursachung des Klimaproblems beigetragen haben und unter anderem auch dadurch heute über ein höheres Wohlstandsniveau verfügen, wird für die Bekämpfung des Klimawandels eine Führungsverantwortung zugewiesen, während den ärmeren Ländern das Recht auf Entwicklung zugebilligt wird. Gleichzeitig sollen die alten Industriestaaten die Entwicklungsund Schwellenländer bei einer möglichst nachhaltigen und klimafreundlichen Gestaltung ihrer Entwicklung unterstützen (ebd.). Dieser Konsens ist bereits in Artikel 3 der 1992 auf dem sogenannten Erdgipfel in Rio de Janeiro verabschiedeten Klimarahmenkonvention der Vereinten Nationen (UNFCCC) festgehalten. Dort heißt es: „Die Vertragsparteien sollen auf der Grundlage der Gerechtigkeit und entsprechend ihren gemeinsamen, aber unterschiedlichen Verantwortlichkeiten und ihren jeweiligen Fähigkeiten das Klimasystem zum Wohl heutiger und künftiger Generationen schützen.“ Ist das Prinzip der ,gemeinsamen, aber unterschiedlichen Verantwortlichkeiten" auch von allen Staaten anerkannt, hat die konkrete Auslegungen dieses Artikels zu zahlreichen Diskussionen geführt, die noch weiter anhalten bzw. wieder neu an Virulenz gewinnen. Der vorliegende Beitrag konzentriert sich daher auf dieses dritte zentrale Feld klimaethischer Erwägungen, der Frage nach der Gerechtigkeit beim Klimaschutz - obgleich eine politische Lösung ohne die Berücksichtigung der anderen ethischen Dimensionen kaum vorstellbar ist.

\section{„Gemeinsame, aber unterschiedliche Verantwortlichkeiten“ vor dem Hinter- grund neuer wirtschaftlicher Realitäten}

Auf der VN-Klimakonferenz im mexikanischen Cancún 2010 (Conference of the Parties - COP 16) wurde erstmals in einem offiziell beschlossenen Verhandlungstext - und nicht nur in einem protokollarisch zur Kenntnis genommenen Dokument wie noch in Kopenhagen (COP 15) - das Ziel der Begrenzung des anthropogenen Klimawandels auf $2^{\circ} \mathrm{C}$ verankert. Dies kann als Konkretisierung des in Artikel 2 der Klimarahmenkonvention formulierten Ziels gelten, ,eine gefährliche anthropogene Störung des Klimasystem“" zu verhindern. Dieses Globalziel wurde aber nicht in ein 
operationalisierbares System der Lastenteilung bei den Anstrengungen zur Reduktion der Treibhausgasemissionen eingebettet, und alle relevanten Bindeglieder zwischen dem globalen Temperaturziel und nationalen Klimaschutzzielen wurden in der Cancún-Vereinbarung ausgeklammert (Pilardeaux/Schulz 2011). Auch ein Jahr später auf der Klimakonferenz in Durban (COP 17) wurde diese Lücke nicht geschlossen. Die globale Lastenteilung bleibt damit abstrakt. Auch das in Artikel 3 der UNFCCC formulierte Prinzip der, gemeinsamen, aber unterschiedlichen Verantwortlichkeiten" bietet allenfalls grobe Orientierung. Aufbauend auf diesem Prinzip sind in Annex I der Klimarahmenkonvention diejenigen Staaten aufgelistet, die als entwickelte Länder gelten. Alle anderen, dort nicht genannten Staaten gelten als Entwicklungsländer. Diese strukturelle Ausformung des Prinzips der ,gemeinsamen, aber unterschiedlichen Verantwortlichkeiten" führte im Rahmen des KyotoProtokolls in eine Aufteilung der Welt in Staaten mit und Staaten ohne rechtlich bindende Klimaschutzverpflichtungen.

Diese Aufteilung wird, vor allem aufgrund der neuen wirtschaftlichen und geopolitischen Realitäten, die sich seit der Verabschiedung der Konvention 1992 herausgebildet haben, von einer wachsende Gruppe von Staaten in Frage gestellt zuletzt bei den Diskussionen um die zweite Verpflichtungsperiode des Kyoto-Protokolls beim Klimagipfel in Durban von Kanada, Japan, Russland und nicht mehr nur den USA. Die Anhänge der Konvention wurden seit Inkrafttreten nur minimal geändert, so dass aufstrebende Schwellenländer wie China oder Indien, aber auch jüngere OECD-Länder wie Mexiko und Südkorea nicht im Annex I genannt sind. Aber nicht nur die wirtschaftliche Leistungsfähigkeit dieser Länder hat in den vergangenen 20 Jahren stark zugenommen, sondern auch ihrer Bedeutung als Verursacher des Klimawandels. China hat im Jahr 2009 die USA als Hauptemittent an Treibhausgasen überflügelt und die kumulativen Emissionen der sogenannten Entwicklungsländer übertreffen mittlerweile den Treibhausgasausstoß der im Annex I gelisteten Länder mit Klimaschutzverpflichtungen. Bleiben die durchschnittlichen pro Kopf-Emissionen dieser Länder auch vergleichsweise gering, beheimaten sie in der Summe mittlerweile eine ebenso große Anzahl an Konsumenten, die im gleichen - und zum Teil höheren - Ausmaß für den Ausstoß von Treibhausgasen verantwortlich sind, wie die Konsumenten der frühindustrialisierten Nationen (Harris 2010: $127 \mathrm{f}$.). 


\section{Was wird geteilt: Emissionsrechte oder Minderungsanstrengungen?}

Es wird vielfach versucht, die ,unterschiedlichen Verantwortlichkeiten“ der Staaten anhand von Kriterien zu quantifizieren, die Gesamtverantwortung also aufzuteilen. Dabei ist zunächst zu klären, was es überhaupt gerecht aufzuteilen gilt. Grundsätzlich lassen sich hier zwei Varianten unterscheiden: Einerseits die Aufteilung der gemeinsamen Ressource, das verbleibende Budget an $\mathrm{CO}_{2}$-Emissionen, um einen gefährlichen Klimawandel zu vermeiden, und andererseits die Aufteilung der notwendigen Anstrengung („efforts“) zur Erreichung eines vereinbarten Klimaziels.

Ein Beispiel für die Aufteilung der gemeinsamen Ressource anhand des pro KopfPrinzips ist der sogenannte Budgetansatz des Wissenschaftlichen Beirats der Bundesregierung Globale Umweltveränderungen (WBGU 2009). Aufbauend auf Berechnungen von Klimawissenschaftlern (Meinshausen u. a. 2009) kommt der WBGU zu dem Schluss, dass bis zum Jahr 2050 noch maximal 750 Mrd. Tonnen $\mathrm{CO}_{2}$ aus fossilen Quellen emittiert werden dürfen, wenn die $2^{\circ} \mathrm{C}$-Leitplanke zumindest mit einer 2/3-Wahrscheinlichkeit eingehalten werden soll. Der WBGU schlägt vor, den Staaten Anteile dieses Gesamtbudgets gemäß ihrem Anteil an der Weltbevölkerung zuzuteilen. Die sich daraus ergebenden nationalen Budgets wären für die Hochemissionsländer relativ zeitnah aufgebraucht, während viele ärmere Länder noch über erhebliche Spielräume verfügten. Mittels eines globalen Emissionshandels ließe sich - nach Vorstellung des WBGU - dieses Ungleichgewicht kompensieren und die Hochemissionsländer könnten für Finanzhilfen beim Klimaschutz und der Klimaanpassung Verschmutzungszertifikate von Ländern mit niedrigen Emissionen erwerben. Die Budgets aus diesem Ansatz erlauben jedem Land bis 2050 durchschnittliche pro Kopf-Emissionen von 2,7 $\mathrm{t} \mathrm{CO}_{2}$ - dies wird aber bereits heute von vielen Ländern überschritten, die nicht in Annex I der UNFCCC vertreten sind (z. B. China, Mexiko, Argentinien), in der Regel mit steigendem Emissionstrend - diese Länder wären also zu erheblichen Klimaschutzmaßnahmen gezwungen bzw. müssten Emissionszertifikate zukaufen.

Pro Kopf-Ansätze werden mit Verweis auf Gerechtigkeitsaspekte kritisiert: Zum einen monieren Kritiker, dass Emissionen - abhängig vom Entwicklungsstand und der Abhängigkeit von fossilen Brennstoffen in der jeweiligen Gesellschaft - eine sehr unterschiedliche Rolle spielen können (Gardiner 2004: 584). Ist ein bestimmtes Emissionsniveau an einem Ort zum bloßen Überleben notwendig, ist mit Emissionen in gleicher Höhe an einem anderen Ort ein Leben in Luxus möglich. Zum anderen erlauben es pro Kopf-Budgets, die für Staaten berechnet werden, dass sich insbesondere in Staaten, die durch ein hohes Maß an sozialer Ungleichheit gekennzeichnet sind - Hochemittenten hinter den vergleichsweise moderaten nationalen 
Durchschnittsemissionen „verstecken“ können (Harris 2010: 128). Ein Beispiel hierfür ist China: Bilden die durchschnittlich etwas mehr als $8 \mathrm{t} \mathrm{CO}_{2}$ pro KopfEmissionen für Österreich relativ gut die Emissionen nahezu jedes Österreichers ab, ist dies bei den ca. $5 \mathrm{t}$ Jahresmissionen eines Chinesen nicht der Fall. Während mehr als 300 Millionen Chinesen, insbesondere auf dem Land, von weniger als 1,25 Dollar pro Tag leben und einen entsprechend kleinen $\mathrm{CO}_{2}$-Fußabdruck hinterlassen, leben in den urbanen Zentren wie Peking, Shanghai oder Hongkong zig Millionen, deren Ressourcenverbrauch und Emissionen ohne Probleme mit dem westlichen pro Kopf-Niveau mithalten kann und zum Teil noch weit darüber liegt (ebd.).

Ein weiteres Problem einer Ressourcenaufteilung auf pro Kopf-Basis liegt in der Gleichsetzung von Emissionen mit Wohlstand. Setzt man voraus, dass eine Entkopplung der Wirtschaftsleistung vom Treibhausgasausstoß möglich ist, entfällt das Argument für die Notwendigkeit von Emissionen. Entwicklungsländer argumentieren allerdings vielfach, dass es ihnen an Zugang zu den emissionsarmen Technologien fehlt, so dass sie für ihre wirtschaftliche Entwicklung dennoch auf höhere Emissionen angewiesen sind.

Typische Kriterien für die Aufteilung der notwendigen Klimaschutzanstrengungen sind:

A) Das Kriterium der ,respective capabilities“, welches sich nicht allein auf die finanziellen Möglichkeiten der Staaten beziehen muss, sondern ebenso die Fähigkeit zur Transformation (d.h. im Wesentlichen zur Umstrukturierung der Infrastruktur) miteinschließen kann,

B) das „Polluter Pays“-Prinzip, welches bei der Klimaproblematik mit der Schwierigkeit konfrontiert ist, dass ein nicht unerheblicher Teil der heutigen Verschmutzung in der Vergangenheit erfolgt ist, da sich Kohlendioxid in der Atmosphäre akkumuliert, und

C) das Prinzip der historischen Verantwortung, wobei strittig ist, wie weit die historische Verantwortung reicht, sprich, ob auch die Emissionen berücksichtigt werden können bzw. sollen, die ohne Kenntnis des Klimawandels produziert wurden. Ein interessantes Beispiel für die Aufteilung des Minderungsaufwands ist der maßgeblich vom Stockholm Environment Institute (SEI) entwickelte „Greenhouse Development Rights"-Ansatz. Das Konzept der Greenhouse Development Rights (Baer u. a. 2008) geht von einem mit der $2^{\circ} \mathrm{C}$-Grenze verträglichen Emissionspfad aus - aufgeteilt auf die Staaten werden jedoch nicht Emissionsrechte, sondern Minderungspflichten, das heißt die Abweichung des angestrebten Emissionspfades vom erwarteten „,business as usual“--Pfad. Basis für eine Lastenteilung ist ein Index, der das Verursacherprinzip mit dem Leistungsfähigkeitsprinzip kombiniert. Ausgehend vom Recht auf Entwicklung wird ein „Entwicklungsschwellenwert“ bei einer Ein- 
kommensgrenze von jährlich 7.500 US-Dollar definiert. Menschen, deren Einkommen unterhalb dieser Schwelle liegt, wird zugestanden, dass sie Entwicklung als Priorität haben - sie sind also nicht für den Klimaschutz verantwortlich. Für Menschen, deren Einkommen oberhalb der Schwelle liegt, wird dagegen angenommen, dass sie Verantwortung dafür tragen, dass andere ihr Recht auf Entwicklung ebenfalls realisieren können. Der „Leistungsfähigkeitsindex“ eines Staates wird schließlich aus dem ab 1990 kumulierten pro Kopf-Einkommen der Bevölkerung oberhalb von 7.500 US-Dollar bestimmt. Analog wird der Index für die Verursachung abgeleitet aus den seit 1990 kumulierten pro Kopf-Emissionen abzüglich der Emissionen, die mit dem Konsum unterhalb der 7.500 US-Dollar-Schwelle verbunden sind. Der Gesamtindex (Global Responsibility and Capability Index, RCI) kombiniert die beiden Teilindizes zu gleichen Teilen. Länder werden in diesem Ansatz nicht länger als black box betrachtet und lediglich anhand ihres nationalen Durchschnittseinkommens oder -emissionswertes unterschieden. Stattdessen wird die ungleiche Einkommens- und Emissionsverteilung berücksichtigt. Ein so definierter Index könnte im Rahmen der internationalen Klimapolitik unterschiedlich operationalisiert werden: beispielsweise ließe er sich als Einzahlungsschlüssel für einen globalen Klimaschutzfonds verwenden, oder als Schlüssel für die Anteile der Staaten an einem global definierten relativen Minderungsziel, das heißt einer Reduktion gegenüber der „business as usual“-Entwicklung. Der „Greenhouse Development Rights“-Ansatz bietet damit einen Schlüssel zur Lastenteilung an, der nicht nur die Gerechtigkeit zwischen Ländern, sondern auch die Gerechtigkeit innerhalb von Ländern berücksichtigt und damit die oben skizzierten, zentralen Schwächen staatszentrierter pro Kopf-Ansätze bzgl. des Emissionsbudgets überwindet. Problematisch an den „Greenhouse Development Rights“ - wie grundsätzlich an allen Konzepten, die den Aufwand der Minderung verteilen - ist dagegen, dass sie auf Prognosen einer zukünftigen, fiktiven Entwicklung angewiesen sind: Um zu wissen, wie viel Aufwand erforderlich ist, um vom ,normalen“ Pfad abzuweichen, ist eine Vorstellung dessen notwendig, was ohne diesen Aufwand passieren würde - der ,business as usual“Pfad. Dieser ist hochgradig unsicher, und auch im Nachhinein nicht verifizierbar, da er ja gerade eben nicht beschritten werden soll.

\section{Kosmopolitische Perspektiven: Staaten und Weltbürger tragen Verantwor- tung für den Schutz der globalen Allmende}

Unabhängig davon, ob sich die Aufteilung der Lasten an der gemeinsamen Ressource oder den notwendigen Minderungsanstrengungen orientiert, ist den verschiedenen Ansätzen gemeinsam, dass die Verantwortung für ein stabiles Klima- 
system für viele Industrieländer höher ist als sie durch Klimaschutz in ihrem eigenen Land erreichen können. Daraus ergibt sich die ethische „Verpflichtung“, anderen, weniger entwickelten Staaten beim Klimaschutz zur Seite zu stehen. Die Erkenntnis, dass die Einhaltung eines Temperaturziels nur durch eine absolute Begrenzung der kumulierten Emissionen erreicht werden kann, macht die Verhandlungen um das burden sharing zu einem Nullsummenspiel: Legt ein Staat sich auf einen Emissionspfad fest, bestimmt er damit automatisch den Spielraum, der den anderen Staaten noch bleibt. Kriterienbasierte Konzepte für eine internationale Lastenteilung beim Klimaschutz, wie sie oben dargelegt worden sind, scheinen daher geradezu geboten.

In den VN-Klimaverhandlungen sind solche Konzepte bisher allenfalls sehr kontrovers diskutiert worden, und für die nächsten Jahre werden sie kaum eine Rolle spielen. Nach den Verhandlungen in Durban im Dezember 2011 steht fest, dass ein gemeinsames, für alle Staaten geltendes neues Abkommen erst für die Zeit nach 2020 ausgehandelt wird. Bis dahin wird der Klimaschutz faktisch nach einem Verfahren betrieben, bei dem Staaten individuell entscheiden, wie viel Klimaschutz sie in ihrem eigenen Land betreiben und wie viel finanzielle Mittel sie für andere Staaten bereitstellen möchten. Die Rolle des internationalen Prozesses ist darauf reduziert, die gemeldeten Ziele und ihre Erreichung einer mehr oder minder ausführlichen Überprüfung zu unterziehen; ein Mandat, die jeweiligen Angebote - nach welchen Kriterien auch immer - individuell zu bewerten, existiert nicht. Bis zum Jahr 2020 ist ein quantifizierbares, kriterienbasiertes, international abgestimmtes System der Lastenverteilung damit vom Tisch.

Paul G. Harris hat in seiner Erörterung der Frage der Klimagerechtigkeit darauf hingewiesen, dass die Klimaproblematik inhärent eine kosmopolitische Perspektive evoziert: „There is no American or Chinese climate system (as distinguished from weather systems); there is only one atmosphere, and every person contributes to changes in global climate, albeit with varying effect in different places, regardless of where he is located. Obligations - for states and for people - arise from this circumstance" (Harris 2010: 152). Mit anderen Worten, nur weil Staaten sich nicht auf die notwendigen Maßnahmen einigen können, um die globale Allmende eines stabilen Klimas zu schützen, kann dies nicht bedeuten, dass einzelne Hochemittenten in den jeweiligen Staaten von ihrer Verantwortung für den Klimaschutz entbunden sind (ebd.: 187). Da Menschen unabhängig von ihrem Ort dazu in der Lage sind, durch ihr Verhalten zur weiteren Erderwärmung beizutragen, seien sie ebenfalls - und nicht nur Staaten - Träger bezogener Rechte, Verantwortlichkeiten und Pflichten. Durch diese Ausweitung der Diskussion um internationale Klimagerechtigkeit, die sich bislang vor allem auf die Verantwortung von Nationalstaaten bezog (ebd.: 10), rückt auch die wachsende Bedeutung der neuen Konsumenten in den 
aufstrebenden Schwellenändern in den Fokus, denen bislang kaum eine Verantwortung für den Klimaschutz zugeschrieben worden ist. Diese wachsende Gruppe an Verbrauchern mit einem emissionsintensiven Lebensstil in die internationalen Klimaschutzbemühungen mit einzubinden, ist nicht nur eine Frage der Gerechtigkeit, sondern schiere Notwendigkeit, um jedes auch nur halbwegs ambitionierte Klimaziel zu erreichen.

\section{Literatur}

Baer, Paul/Athanasiou, Tom/Kartha, Sivan/Kemp-Benedict, Eric, 2008: The Greenhouse Development Rights Framework - The right to development in a climate constrained world, Revised second edition, Berlin.

Broome, John, 2008: The Ethics of Climate Change, in: Scientific American (June Issue), 69-73.

Gardiner, Stephen M., 2004: Ethics and Global Climate Change, in: Ethics 114 (April Issue), 555-600.

Harris, Paul G., 2010: World Ethics and Climate Change. From International to Global Justice, Edinburgh.

Meinshausen, Malte/Meinshausen, Nicolai/Hare, William/Raper, Sarah C. B./Frieler, Katja/Knutti, Reto/Frame, David J./Allen, Myles R., 2009: Greenhouse gas emission targets for limiting global warming to $2^{\circ} \mathrm{C}$, in: Nature 458 (No. 7242), 1158-1162.

Pilardeaux, Benno/Schulz, Astrid, 2011: Klimaschutz: neue internationale Allianzen, in: Günter Altner/Heike Leitschuh/Gerd Michelsen/Udo E. Simonis/Ernst Ulrich von Weizsäcker (Hrsg.), Grüner Umbau. Neue Allianzen für die Umwelt. Jahrbuch Ökologie 2012, Stuttgart, 90-98.

Rogelj Joeri/Hare, William/Lowe, Jason/van Vuuren, Detlef P./Riahi, Keywan/ Matthews, Ben/Hanaoka, Tatsuya/Jiang, Kejun/Meinshausen, Malte, 2011: Emission pathways consistent with a $2{ }^{\circ} \mathrm{C}$ global temperature limit, in: Nature Climate Change 1258 (November Issue), 21.

Sommer, Bernd, 2011: Interdependenzen und Ungleichzeitigkeiten im Kontext des anthropogenen Klimawandels, in: Leviathan 39 (1), 55-72.

Wissenschaftlicher Beirat der Bundesregierung Globale Umweltveränderungen $(W B G U)$, 2009: Kassensturz für den Weltklimavertrag - Der Budgetansatz, Berlin. 
Korrespondenzanschrift

Dr. Astrid Schulz

Wissenschaftlicher Beirat der Bundesregierung Globale Umweltveränderungen (WBGU)

Luisenstraße 46

10117 Berlin

E-Mail: aschulz@wbgu.de

Dr. Bernd Sommer

Interdisziplinäres Institut für Umwelt-, Sozial- und Humanwissenschaften Universität Flensburg

Auf dem Campus 1

24943 Flensburg

E-Mail: bernd.sommer@uni-flensburg.de 